\title{
Performance of Three Measures of Comorbidity in Predicting Critical COVID- 19: A Retrospective Analysis of 4607 Hospitalized Patients
}

\author{
David Monterde, (iD) ${ }^{1,2}$ \\ Gerard Carot-Sans, (D) ${ }^{2,3}$ \\ Miguel Cainzos-Achirica, ${ }^{4,5}$ \\ Sònia Abilleira, (D) ${ }^{1,6}$ \\ Marc Coca, ${ }^{2,3}$ Emili Vela, ${ }^{2,3}$ \\ Montse Clèries, ${ }^{2,3}$ \\ Damià Valero-Bover, 2,3 \\ Josep Comin-Colet, ${ }^{7-9}$ \\ Luis García-Eroles, ${ }^{2,3}$ \\ Pol Pérez-Sust, (iD) ${ }^{3}$ \\ Miquel Arrufat, (1D 1 \\ Yolanda Lejardi, ' \\ Jordi Piera-Jiménez $\mathbb{D}^{2,3,10}$
}

'Catalan Institute of Health, Barcelona, Spain; ${ }^{2}$ Digitalization for the Sustainability of the Healthcare System (DS3), Sistema de Salut de Catalunya, Barcelona, Spain; ${ }^{3}$ Servei Català de la Salut, Barcelona, Spain; ${ }^{4}$ Center for Outcomes Research, Houston Methodist, Houston, TX, USA; 5 Johns Hopkins Ciccarone Center for the Prevention of Cardiovascular Disease, Johns Hopkins Medical Institutions, Baltimore, MD, USA; ${ }^{6}$ CIBER Epidemiología y Salud Pública (CIBERESP), Barcelona, Spain; 'Department of Cardiology, Hospital Universitari de Bellvitge, L'Hospitalet de Llobregat, Barcelona, Spain; ${ }^{8}$ Bioheart-

Cardiovascular Diseases Research Group (Idibell), L'Hospitalet de Llobregat, Barcelona, Spain; ${ }^{9}$ Department of Clinical Sciences, School of Medicine, Universität de Barcelona - UB, L'Hospitalet de Llobregat, Barcelona, Spain; ${ }^{10}$ Open Evidence Research Group, Universitat Oberta de Catalunya, Barcelona, Spain

Correspondence: Jordi Piera-Jiménez Servei Català de la Salut (CatSalut) Travessera de les Corts, I3I-159 (Edifici Olímpia), Barcelona, 08028, Spain

Tel +34634283II0

Email jpiera@catsalut.cat
Background: Comorbidity burden has been identified as a relevant predictor of critical illness in patients hospitalized with coronavirus disease 2019 (COVID-19). However, comorbidity burden is often represented by a simple count of few conditions that may not fully capture patients' complexity.

Purpose: To evaluate the performance of a comprehensive index of the comorbidity burden (Queralt DxS), which includes all chronic conditions present on admission, as an adjustment variable in models for predicting critical illness in hospitalized COVID-19 patients and compare it with two broadly used measures of comorbidity.

Materials and Methods: We analyzed data from all COVID-19 hospitalizations reported in eight public hospitals in Catalonia (North-East Spain) between June 15 and December 8 2020. The primary outcome was a composite of critical illness that included the need for invasive mechanical ventilation, transfer to ICU, or in-hospital death. Predictors including age, sex, and comorbidities present on admission measured using three indices: the Charlson index, the Elixhauser index, and the Queralt DxS index for comorbidities on admission. The performance of different fitted models was compared using various indicators, including the area under the receiver operating characteristics curve (AUROCC).

Results: Our analysis included 4607 hospitalized COVID-19 patients. Of them, 1315 experienced critical illness. Comorbidities significantly contributed to predicting the outcome in all summary indices used. AUC $(95 \%$ CI) for prediction of critical illness was 0.641 (0.624-0.660) for the Charlson index, $0.665(0.645-0.681)$ for the Elixhauser index, and 0.787 (0.773-0.801) for the Queralt DxS index. Other metrics of model performance also showed Queralt DxS being consistently superior to the other indices.

Conclusion: In our analysis, the ability of comorbidity indices to predict critical illness in hospitalized COVID-19 patients increased with their exhaustivity. The comprehensive Queralt DxS index may improve the accuracy of predictive models for resource allocation and clinical decision-making in the hospital setting.

Keywords: comorbidity, multimorbidity, COVID-19, hospitalization, risk

\section{Introduction}

During 2020, the rapid spread of the severe acute respiratory syndrome coronavirus 2 (SARS-CoV-2) and the severity of coronavirus disease 2019 (COVID-19) led to the collapse of many healthcare systems worldwide, particularly hospital and intensive care unit (ICU) resources. Recently approved vaccines against SARS-CoV-2 infection are expected to ease hospital resources pressure. ${ }^{1}$ Nevertheless, in many settings, COVID-19 continues to cause high hospital demand, which requires adequate healthcare provision 
planning, particularly in scenarios of a shortage of resources or overburdening of hospitals.

Early in the pandemic, underlying comorbidities were pointed out as significant prognostic factors for the development of severe illness. Initial analyses of a large series of COVID-19 patients revealed that hospitalized patients with chronic conditions like diabetes, hypertension, chronic obstructive pulmonary disease, or cardiovascular diseases were more likely to develop severe COVID-19, with no consensus regarding the contribution of each comorbidity to explaining differences in COVID-19 outcomes. $^{2-5}$ Based on this evidence, the patient's comorbidity burden on admission has been included in many models for predicting hospital outcomes in COVID-19 patients, sometimes combined with post-admission information (eg, vital signs, laboratory and imaging results). ${ }^{6}$ However, the comorbidity burden is typically represented by a simple count of few chronic conditions (ie, up to 12) that does not fully capture patients' complexity. ${ }^{7-14}$

Another approach to representing the patient's comorbidity burden is using a comorbidity index that combines information about several comorbidities into a single score. ${ }^{17,18}$ This approach yields more complex models with a more challenging interpretation; however, it allows adjusting for multiple factors, likely improving model accuracy. ${ }^{18}$ Known examples of comorbidity indices include the Charlson Comorbidity Index ${ }^{15}$ and the Elixhauser index for hospitalized patients. ${ }^{16}$ In 2020, we developed a comprehensive risk index tool for hospitalized patients, the Queralt Index, which includes a measure of pre-existing comorbidities (Queralt DxS). ${ }^{19}$ The Queralt DxS combines and weighs more than 2100 relevant acute and chronic diagnostic codes. Queralt DxS has shown optimal performance for risk adjustment when measuring comorbidity burden in hospitalized patients. ${ }^{19}$

Understanding the risk factors, such as comorbidities associated with hospital outcomes in COVID-19 patients, is crucial for healthcare planning during future waves of the infection. However, most predicting models published to date rely on simple counts of comorbidities, and no studies have explored the appropriate measure for adjusting for multimorbidity in the COVID-19 setting. In this study, we assessed the performance of Queralt DxS as a comorbidity measure in models for predicting outcomes in hospitalized COVID-19 patients and compared it with other widely used comorbidity measures: the Charlson and Elixhauser indices.

\section{Materials and Methods Data Sources}

In this retrospective analysis, we included data from patients hospitalized due to COVID-19 in any of the eight hospitals of the Catalan Institute of Health (ICS), the leading health care provider in Catalonia (North-East Spain; 7.5 million inhabitants). The ICS provides universal health care to nearly $70 \%$ of the Catalan population, and accounts for approximately $30 \%$ of all hospitalizations reported in Catalonia. ICS hospitals systematically collect and store data on diagnoses and resource utilization into a centralized database. We screened the ICS database for all admissions due to COVID-19 occurred within the investigated period, which was restricted to June 15-December 8, 2020 , to prevent biases associated with the unprecedented hospital overburdening experienced in Spain during the first wave of the COVID-19 outbreak. $^{20,21}$ The database was locked on March 30, 2021.

Hospital admissions due to COVID-19 were identified according to the following codes of the International Classification of Diseases, 10th Revision, Clinical Modification (ICD-10-CM) system: B97.29, B97.21, B34.2, J12.81, J12.89, and the recently added code for labconfirmed COVID-19 U07.1. We included all hospital admissions of patients who either died in the hospital or were discharged home. Records from patients transferred from or to other hospitals were excluded from the analysis to ensure the completeness of clinical data during the full index hospitalization.

All data were handled according to the General Data Protection Regulation 2016/679 on data protection and privacy for all individuals within the European Union and the local regulatory framework regarding data protection. The independent ethics committee of the Bellvitge Biomedical Research Institute (IDIBELL) approved the study protocol (Ref. PR195/21) and waived the need for informed consent, as the data were generated as part of routine clinical care and fully de-identified for analytic purposes.

\section{Outcomes and Predictors}

The study outcome ("critical illness" in patients hospitalized with COVID-19) was a composite that included the need for invasive mechanical ventilation, transfer to the intensive care unit (ICU), or in-hospital death. The need for invasive mechanical ventilation was identified by the presence of any of the following procedure codes recorded in the hospital database for billing purposes: 5A09357, 
5A09457，5A09557，5A1935Z，5A1945Z，5A1955Z, 09HN7BZ, 09HN8BZ, 0BH13EZ, 0BH17EZ, 0BH18EZ, and $0 \mathrm{CHY} 7 \mathrm{BZ}$.

Potential predictors considered for the analysis included age, sex, and comorbidities present on admission. The latter were measured using three multi-comorbidity indices: the Charlson Comorbidity Index, the Elixhauser index, and Queralt DxS. In another model, we also used the 27 comorbidities included in the Elixhauser index, each of them separately. The Charlson and Elixhauser indices were estimated using the ICD-10 coding system proposed by Quan et al. ${ }^{22}$ For the Charlson index, diagnostic weights were assigned based on the original formulation by Charlson et al. ${ }^{15}$ For the Elixhauser index, we used the weights proposed by Moore et al. ${ }^{23}$ Queralt DxS belongs to a family of three indices for predicting clinical outcomes in hospitalized patients that provide a numerical value from the weighted sum of diagnoses (primary, secondary present on admission, and complications) from a list of 2119 diagnostic code groups. ${ }^{19}$ In this analysis, we used an updated version of the index (version 6.0), computed using healthcare data recorded in the routine care setting between 2018 and 2019.

\section{Statistics}

Data on demographic and clinical variables and outcomes were described as frequency and percentage, mean and standard deviation (SD), and/or median and interquartile range (IQR, defined as the 25th and 75th percentiles), as appropriate. The Charlson and Elixhauser indices were computed using the Comorbidity library by Gasparini, ${ }^{24}$ Queralt DxS was generated using R functions and instructions provided in the Supplementary File. For descriptive purposes only, patients were also stratified at four risk levels (ie, low, moderate, high, and very high) based on the 50th, 80th, and 95th percentiles of Queralt DxS, as previously described. ${ }^{19}$

Since the primary objective was to compare the performance of each index of comorbidity when added to a given multivariate risk prediction model, we built five logistic regression models to predict the composite outcome of critical illness: a baseline model with age (introduced into the model as a continuous variable) and sex, three models including the baseline and each of the comorbidity measures (ie, Charlson index, Elixhauser index, and Queralt DxS), and one including the 27 diagnoses included in the Elixhauser index separately. The performance of each model was evaluated using five statistical measures of model performance: the deviance, the Akaike information criterion (AIC), ${ }^{25}$ the Bayesian Information Criterion (BIC) ${ }^{26}$ the area under the receiver operating characteristic curve (AUROCC), and the area under the precisionrecall curve (AUPRC). ${ }^{27}$ The $95 \%$ confidence intervals (CI) of AUROCC were estimated using 1000 bootstrap samples and confirmed with the DeLong criteria. ${ }^{28}$ All analyses were performed using the $\mathrm{R}$ statistical package (version 4.0.3). ${ }^{29}$

\section{Results \\ Study Population}

Between June 15 and December 8, 2020, 4607 patients were admitted to the ICS hospitals with COVID-19. Table 1 summarizes the demographic characteristics of the study population according to the incidence of critical illness (ie, need for invasive mechanical ventilation, transfer to ICU, or death) on admission and/or during hospitalization. The corresponding comorbidity burden, measured using the three analyzed indices, is shown in Table 2. Patients who experienced critical illness on/after admission were significantly older and had higher scores of comorbidity burden irrespective of the index used. Men experienced critical illness more frequently.

According to the distribution of patients across the five risk levels of Queralt DxS, 2145 (46.6\%) patients were at low risk, $1509(32.8 \%)$ at moderate risk, $730(15.8 \%)$ at high risk, and $223(4.8 \%)$ at very high risk. Figure 1 shows the distribution of patients with and without critical illness, according to age, sex, and Queralt risk group.

\section{Performance of Comorbidity Indices for Prediction of Critical COVID-19 IIIness}

Table 3 shows the overall performance of each model, computed using various measures of the magnitude of the effect. The addition of a multimorbidity measure to the baseline model increased the AUROCC and AUPRC values of the model, which were consistent with all other metrics of model performance. In all, the best performance was consistently observed for the model that included age, sex, and Queralt DxS (Table 3). Figure 2 shows the ROC and precision-recall curves of the five models explored.

In the three models including a comorbidity index, this factor significantly contributed to predicting critical illness, irrespective of the comorbidity index used in the model (Figure 3). In the model including Queralt $\mathrm{DxS}$ as a comorbidity measure, age groups lost statistical significance. 
Table I Demographic Characteristics of Patients Included in the Analysis, Grouped by Incidence of Critical Illness During the Index Hospitalization $^{\mathrm{a}}$

\begin{tabular}{|c|c|c|c|c|}
\hline & $\begin{array}{l}\text { Without Critical IIIness } \\
\qquad(\mathrm{N}=3292)\end{array}$ & Critical Illness $(N=13 \mid 5)$ & Total $(\mathrm{N}=4607)$ & $p$ value \\
\hline $\begin{array}{l}\text { Age } \\
\qquad \text { Mean (SD) } \\
\text { Median (QI, Q3) }\end{array}$ & $\begin{array}{c}58.2(19.7) \\
59.0(45.0,73.0)\end{array}$ & $\begin{array}{c}66.1(17.5) \\
68.0(56.0,79.0)\end{array}$ & $\begin{array}{c}60.5(19.4) \\
62.0(48.0,75.0)\end{array}$ & $<0.001$ \\
\hline $\begin{array}{l}\text { Age group } \\
0-4 \\
5-9 \\
10-14 \\
15-19 \\
20-24 \\
25-29 \\
30-34 \\
35-39 \\
40-44 \\
45-49 \\
50-54 \\
55-59 \\
60-64 \\
65-69 \\
70-74 \\
75-79 \\
80-84 \\
85-89 \\
90->\end{array}$ & $\begin{array}{l}42(1.3 \%) \\
11(0.3 \%) \\
8(0.2 \%) \\
32(1.0 \%) \\
65(2.0 \%) \\
108(3.3 \%) \\
144(4.4 \%) \\
181(5.5 \%) \\
195(5.9 \%) \\
260(7.9 \%) \\
305(9.3 \%) \\
303(9.2 \%) \\
316(9.6 \%) \\
297(9.0 \%) \\
275(8.4 \%) \\
271(8.2 \%) \\
190(5.8 \%) \\
160(4.9 \%) \\
129(3.9 \%)\end{array}$ & $\begin{array}{l}6(0.5 \%) \\
4(0.3 \%) \\
3(0.2 \%) \\
8(0.6 \%) \\
5(0.4 \%) \\
13(1.0 \%) \\
25(1.9 \%) \\
36(2.7 \%) \\
47(3.6 \%) \\
62(4.7 \%) \\
96(7.3 \%) \\
122(9.3 \%) \\
146(11.1 \%) \\
126(9.6 \%) \\
155(11.8 \%) \\
143(10.9 \%) \\
129(9.8 \%) \\
95(7.2 \%) \\
94(7.1 \%)\end{array}$ & $\begin{array}{l}48(1.0 \%) \\
15(0.3 \%) \\
11(0.2 \%) \\
40(0.9 \%) \\
70(1.5 \%) \\
121(2.6 \%) \\
169(3.7 \%) \\
217(4.7 \%) \\
242(5.3 \%) \\
322(7.0 \%) \\
401(8.7 \%) \\
425(9.2 \%) \\
462(10.0 \%) \\
423(9.2 \%) \\
430(9.3 \%) \\
414(9.0 \%) \\
319(6.9 \%) \\
255(5.5 \%) \\
223(4.8 \%)\end{array}$ & $<0.001$ \\
\hline $\begin{array}{l}\text { Sex } \\
\qquad \text { Male } \\
\text { Female }\end{array}$ & $\begin{array}{l}1742(52.9 \%) \\
1550(47.1 \%)\end{array}$ & $\begin{array}{l}844(64.2 \%) \\
471(35.8 \%)\end{array}$ & $\begin{array}{l}2586(56.1 \%) \\
2021(43.9 \%)\end{array}$ & $<0.001$ \\
\hline
\end{tabular}

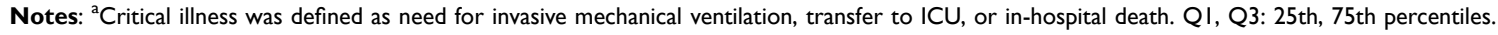
Abbreviation: SD, standard deviation.

\section{Discussion}

Our results using data from the healthcare databases of Catalonia confirm the relevance of comorbidities on admission as a strong predictor of outcomes in patients hospitalized with COVID-19, which is consistent with prior reports. Accordingly, the addition of any of the summary measures of comorbidity burden used in our analysis improved the performance of predictive models compared with age and sex only. Furthermore, for the first time to our knowledge, we showed how different measures of the comorbidity burden can change the accuracy of models for predicting critical illness in hospitalized COVID-19 patients.

In the context of comorbidity burden being a strong predictor of outcomes in COVID-19 patients, we observed that the most comprehensive multi-comorbidity index evaluated, Queralt DxS, showed the strongest contribution to explaining critical illness. Unlike the other two indices investigated (ie, the Charlson and Elixhauser indices), which estimate comorbidity burden from a discrete list of diagnoses, Queralt DxS considers all conditions present on admission among a list of 2119 diagnostic groups. Hence, our findings align with the concerns highlighted by Ording and Sørensen, who warned about residual confounding potentially introduced when underestimating the comorbidity burden by using numerical indices based on rather restrictive definitions of comorbidity. ${ }^{18}$ Remarkably, when introducing an exhaustive summary measure of comorbidities, such as Queralt DxS, to the model, age was no longer a significant predictor. This suggests that the risk of critical illness in older patients is driven by the accumulation of chronic conditions over time rather than by age per se.

In addition to comorbidities, various authors have identified other relevant predictors of hospital outcomes in 
Table 2 Clinical Characteristics of Patients Included in the Analysis, Grouped by Incidence of Critical Illness During the Index Hospitalization $^{\mathrm{a}}$

\begin{tabular}{|c|c|c|c|c|}
\hline & $\begin{array}{l}\text { Without Critical IIIness } \\
\qquad(\mathrm{N}=3292)\end{array}$ & Critical Illness $(\mathrm{N}=|3| 5)$ & Total $(\mathrm{N}=4607)$ & $P$ value \\
\hline $\begin{array}{l}\text { Charlson index } \\
\text { Mean (SD) } \\
\text { Median (QI, Q3) }\end{array}$ & $\begin{array}{c}1.0(1.6) \\
0.0(0.0,1.0)\end{array}$ & $\begin{array}{c}1.6(2.0) \\
1.0(0.0,2.0)\end{array}$ & $\begin{array}{c}1.2(1.7) \\
0.0(0.0,2.0)\end{array}$ & $<0.001$ \\
\hline $\begin{array}{l}\text { Elixhauser index } \\
\text { Mean (SD) } \\
\text { Median (QI, Q3) }\end{array}$ & $\begin{array}{c}1.8(6.5) \\
0.0(-1.0,4.0)\end{array}$ & $\begin{array}{c}5.5(9.2) \\
3.0(-1.0,11.0)\end{array}$ & $\begin{array}{c}2.8(7.6) \\
0.0(-1.0,5.0)\end{array}$ & $<0.001$ \\
\hline $\begin{array}{l}\text { Queralt index (risk) } \\
\text { Mean (SD) } \\
\text { Median (QI, Q3) }\end{array}$ & $\begin{array}{c}17.8(12.2) \\
16.0(9.0,25.0)\end{array}$ & $\begin{array}{c}33.8(18.2) \\
30.0(21.0,42.0)\end{array}$ & $\begin{array}{c}22.3(15.9) \\
20.0(11.0,30.0)\end{array}$ & $<0.001$ \\
\hline $\begin{array}{l}\text { Risk groups } \\
\text { Low } \\
\text { Moderate } \\
\text { High } \\
\text { Very high }\end{array}$ & $\begin{array}{c}1925(58.5 \%) \\
982(29.8 \%) \\
345(10.5 \%) \\
40(1.2 \%)\end{array}$ & $\begin{array}{l}220(16.7 \%) \\
527(40.1 \%) \\
385(29.3 \%) \\
183(13.9 \%)\end{array}$ & $\begin{array}{c}2145(46.6 \%) \\
1509(32.8 \%) \\
730(\mid 5.8 \%) \\
223(4.8 \%)\end{array}$ & $<0.001$ \\
\hline
\end{tabular}

Notes: ${ }^{\mathrm{a}}$ Critical illness was defined as need for invasive mechanical ventilation, transfer to ICU, or in-hospital death. ${ }^{\mathrm{b}}$ The low, moderate, high, and very high risk groups correspond to the 50th, 80th, and 95th percentiles of Queralt DxS in the study population, respectively. QI, Q3: 25th, 75th percentiles.

Abbreviation: SD, standard deviation.

patients with COVID-19, such as inflammatory biomarkers, need for oxygen therapy, and diagnostic images analyzed using advanced machine learning approaches. ${ }^{30-35}$ While these models are helpful for informing clinical decisions based on assessments performed during the hospital stay in individual patients, models that use retrospective information available from electronic health records can provide hospital outcome estimates at the time of hospital admission, thus aiding not only clinicians but also managers and policymakers in hospital resource planning. Our results suggest that all models, irrespective of whether they include data from in-hospital assessments, may benefit from a summary, highly comprehensive measure of the comorbidity burden on admission, such as Queralt DxS. We have made this index and the related code freely available online for research purposes as an open-source tool to facilitate evaluation by other groups either in its current form in other populations or potentially as part of clinical risk estimation tools combining comorbidities and clinical data on admission.
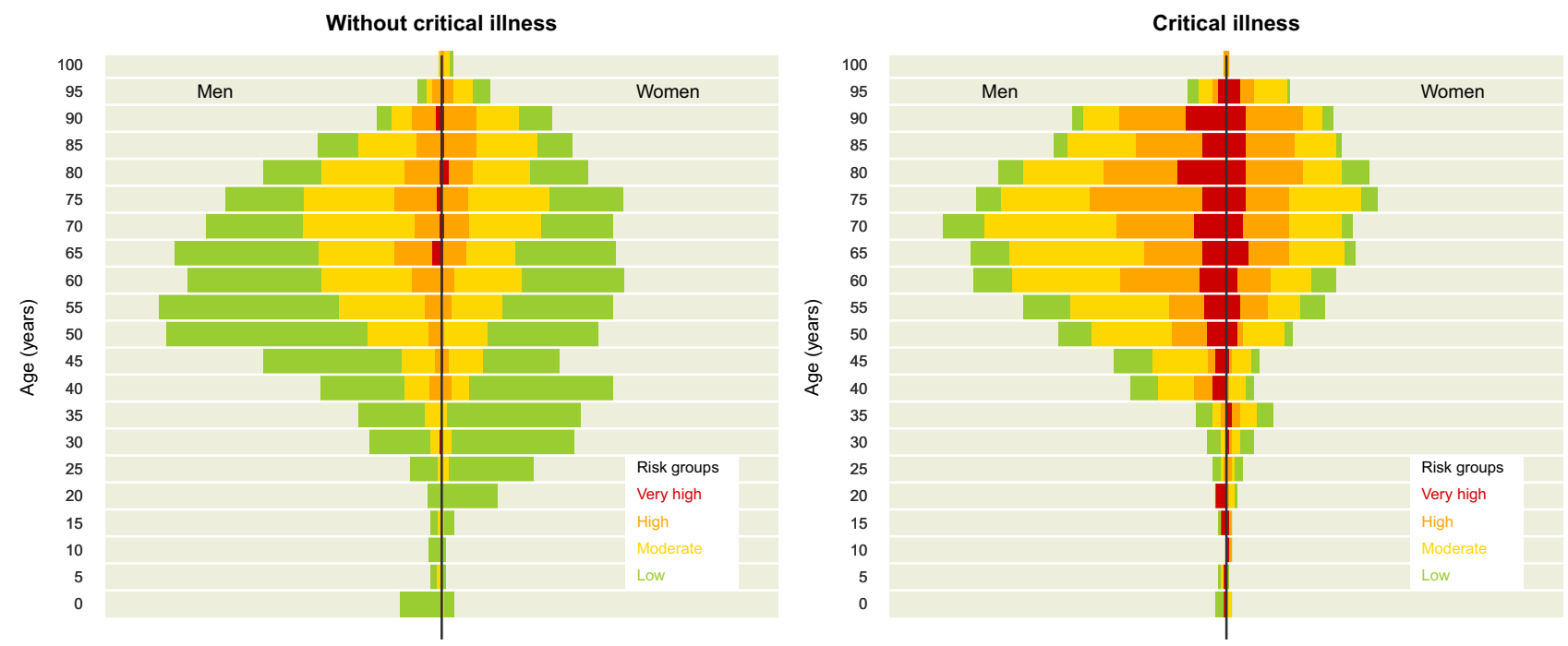

Figure I Participant distribution according to age, sex, and Queralt risk group (corresponding to the 50th, 80th, and 95th percentiles of the Queralt Index). 
Table 3 Performance of the Baseline Model and Models Including Each of the Investigated Measures of Comorbidity

\begin{tabular}{|l|c|c|c|c|c|c|c|c|c|}
\hline Model & Dev & AIC & BIC & AUROCC & \multicolumn{2}{|c|}{ 95\% Cl } & AUPRC & \multicolumn{2}{|c|}{$95 \%$ CI } \\
\hline Age + Sex & 5301 & 5307 & 5326 & 0.632 & 0.614 & 0.650 & 0.387 & 0.366 & 0.415 \\
$\ldots+$ + Charlson & 5265 & 5273 & 5298 & 0.644 & 0.628 & 0.660 & 0.402 & 0.373 & 0.435 \\
$\ldots+$ Elixhauser & 5175 & 5183 & 5208 & 0.667 & 0.650 & 0.683 & 0.441 & 0.415 & 0.465 \\
$\ldots+$ + Queralt & 4501 & 4509 & 4534 & 0.787 & 0.774 & 0.802 & 0.600 & 0.573 & 0.630 \\
\hline
\end{tabular}

Abbreviations: Dev, Deviance (lower values indicate better performance); AIC, Akaike information criteria (lower values indicate better performance); BIC, Bayes criteria (lower values indicate better performance); AUROCC, area under the receiver operating characteristics curve (values range from 0.5 [low discrimination capacity] to I [high discrimination capacity]); AUPRC, area under the precision-recall curve (values range from 0 [low predictive capacity] to I [high predictive capacity]); Cl, confidence interval.

Indices that summarize a high number of variables into a single numerical index, such as Queralt DxS, also have the advantage of allowing more parsimonious models. This is particularly important for machine learning approaches, which may lose performance when increasing the number of variables included in the model. Hence, despite losing sight on the individual effect of each diagnosis, summarizing the information from thousands of pre-
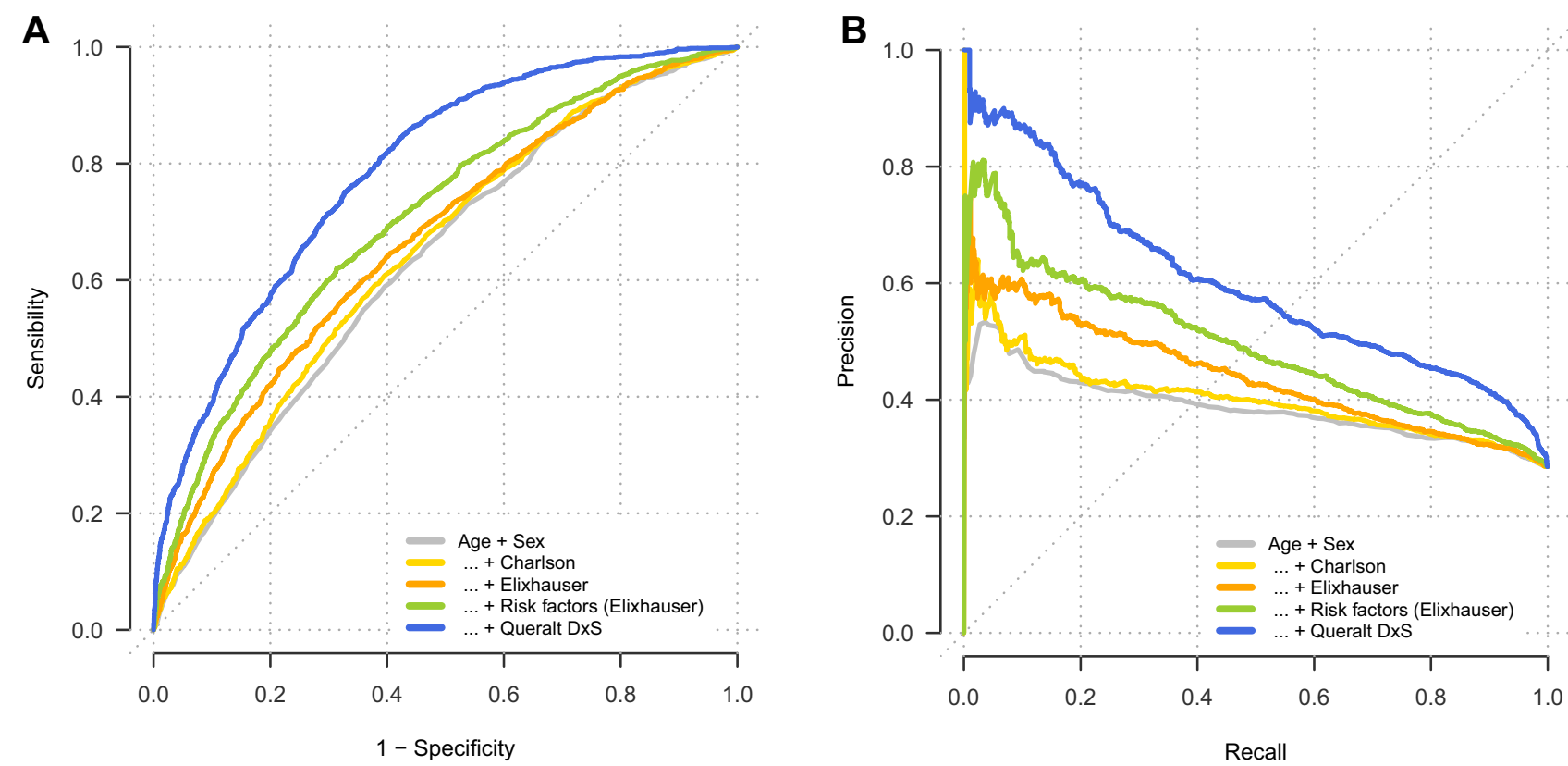

Figure 2 Performance of the five logistic regression models: baseline (age and sex), Charlson (age, sex, and Charlson index), Elixhauser (age, sex, and Elixhauser index), Queralt (age, sex, and Queralt DxS index), and the 27 diagnostic codes included in the Elixhauser index. (A) receiver operating characteristics curve. (B) precision-recall curve.

Age + Sex

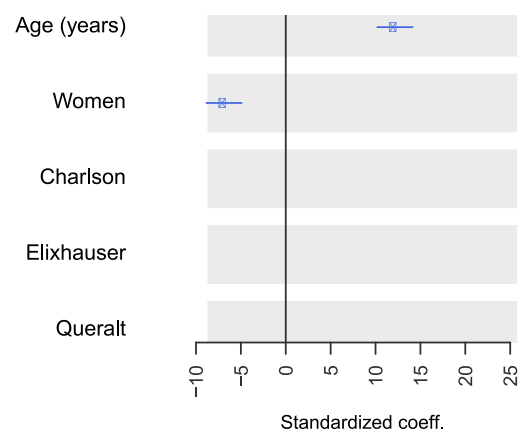

Age + Sex + Charlson

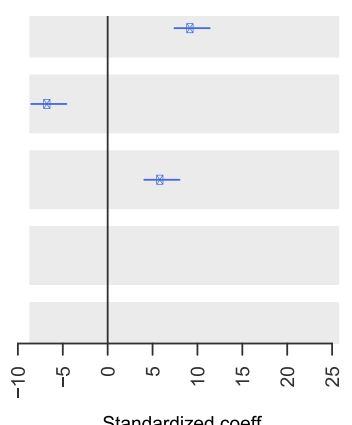

Age + Sex + Elixhauser

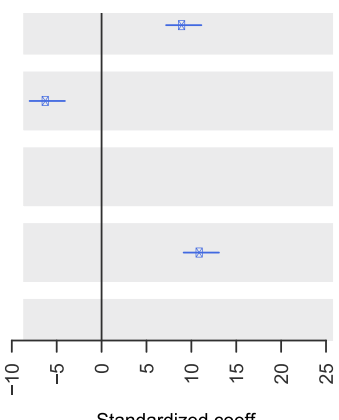

Age + Sex + Queralt DxS

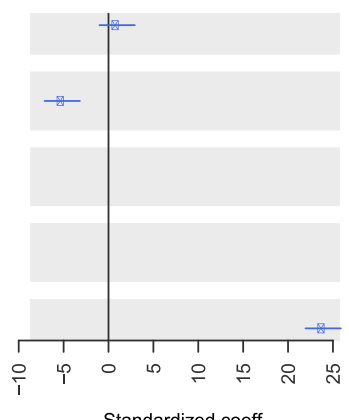

Figure 3 Standardized coefficients according to four logistic regression models: baseline (age and sex), Charlson (age, sex, and Charlson index), Elixhauser (age, sex, and Elixhauser index), and Queralt (age, sex, and Queralt DxS). 
existing diagnoses into a single index that accounts for the relative weights of each one and their prognostic relevance for hospital outcomes may enrich other models while minimizing the risk of overfitting.

\section{Study Limitations}

One of the limitations of Queralt DxS is the lack of validation in settings other than Catalonia; however, we hope that making the software publicly available for research purposes will facilitate external validation moving forward. Moreover, it is worth mentioning that the weights used to estimate the relative contribution of each diagnostic group to health risk were calculated using data collected between 2018 and 2019, before the COVID-19 pandemic. Hence, although the source population of the eight relevant hospitals has remained unchanged since then, the sample of patients admitted with COVID-19 included in this study, and their clinical and sociodemographic profiles do differ from those used when developing the Queralt indices, including Queralt DxS. Therefore, this analysis may be interpreted as a pseudo-validation of Queralt DxS in a Catalan subpopulation hospitalized with COVID-19.

Also, as often occurs in retrospective analyses, our dataset was limited to the data recorded in electronic records during routine care. Nonetheless, the universal coverage of our healthcare system, and the cross-linking of healthcare data from the primary care and specialized settings allowed us to access various sources and very comprehensive health data from our study population and consider all possible diagnoses present on admission, as well as basic demographic data, such as age and sex. Conversely, we were not able to include some preadmission variables such as blood groups, which have been associated with COVID-19 severity $^{36}$ and might modulate the weight of comorbidities when estimating the risk of critical illness, or the SARS-CoV-2 variant, which may influence clinical outcomes of COVID-19 patients. Nevertheless, even without those pieces of information, the performance of Queralt DxS was very robust.

Finally, it is worth mentioning that we did not adjust for treatments administered or the type of care received. COVID-19 is an evolving pandemic, and the development and implementation of pathogenesis-directed therapies ${ }^{37}$ and care pathways ${ }^{38}$ may strongly influence the clinical outcome, regardless of the baseline risk factors of hospitalized patients. Due to the relatively narrow investigated period and early stage of the pandemic (ie, June-
December 2020), we expect the type of care to be homogeneous among study patients. However, future analyses are warranted to investigate how newly developed therapies, vaccination, and improved care pathways influence the weight of the comorbidity burden on predicting critical illness.

\section{Conclusion}

Our findings show that the burden of pre-existing comorbidities significantly improves the prediction of the risk of critical illness in patients hospitalized with COVID-19, particularly when measured exhaustively using a tool such as Queralt DxS. This comorbidity index, which is freely available for research purposes, may improve the accuracy of risk models aimed at supporting clinical decision-making and hospital resource planning in hospitalized patients, including those with COVID-19. Future studies investigating the influence of the comorbidity burden, measured with the Queralt DxS tool, on hospital outcomes in the context of vaccination and improved care pathways are warranted.

\section{Abbreviations}

AIC, Akaike information criterion. AUPRC, area under the precision-recall curve. AUROCC, area under the receiver operating characteristic curve. CI, confidence interval. CM, clinical modification. COVID-19, coronavirus disease 2019. ICD, International Classification of Diseases. ICS, Catalan Institute of Health. ICU, intensive care unit. IQR, interquartile range.

\section{Ethics Approval and Informed Consent}

The study protocol was approved by the independent ethics committee of the Bellvitge Biomedical Research Institute (IDIBELL) (Ref. PR195/21), which waived the need for informed consent, as the data were generated as part of routine clinical care and fully de-identified for analytic purposes.

\section{Acknowledgment}

The authors would like to thank Toni Fuentes for his support in building the dataset used in this analysis.

\section{Author Contributions}

All authors made a significant contribution to the work reported, whether that is in the conception, study design, execution, acquisition of data, analysis and interpretation, 
or in all these areas; took part in drafting, revising or critically reviewing the article; gave final approval of the version to be published; have agreed on the journal to which the article has been submitted; and agree to be accountable for all aspects of the work.

\section{Disclosure}

David Monterde declares that he is the developer of the Queralt System. This tool is available online for research purposes at no cost. The authors report no other conflicts of interest in this work, which did not receive specific funding.

\section{References}

1. Lee P, Kim C-U, Seo SH, Kim D-J. Current status of COVID-19 vaccine development: focusing on antigen design and clinical trials on later stages. Immune Netw. 2021;21(1):e4. doi:10.4110/ in.2021.21.e4

2. Grasselli G, Greco M, Zanella A, et al. Risk factors associated with mortality among patients with COVID-19 in intensive care units in Lombardy, Italy. JAMA Intern Med. 2020:1-11. doi:10.1001/ jamainternmed.2020.3539.

3. Turcotte JJ, Meisenberg BR, MacDonald JH, et al. Risk factors for severe illness in hospitalized Covid-19 patients at a regional hospital. PLoS One. 2020;15(8 August):e0237558. doi:10.1371/journal. pone. 0237558

4. Clark A, Jit M, Warren-Gash C, et al. Global, regional, and national estimates of the population at increased risk of severe COVID-19 due to underlying health conditions in 2020: a modelling study. Lancet Glob Heal. 2020;8(8):e1003-e1017. doi:10.1016/S2214-109X(20) 30264-3

5. Petrilli CM, Jones SA, Yang J, et al. Factors associated with hospital admission and critical illness among 5279 people with coronavirus disease 2019 in New York City: prospective cohort study. BMJ. 2020;369. doi:10.1136/bmj.m1966

6. Wynants L, Van Calster B, Collins GS, et al. Prediction models for diagnosis and prognosis of covid-19: systematic review and critical appraisal. BMJ. 2020;369:26. doi:10.1136/bmj.m1328

7. Huang H, Cai S, Li Y, et al. Prognostic factors for COVID-19 pneumonia progression to severe symptoms based on earlier clinical features: a retrospective analysis. Front Med. 2020;7:557453. doi:10.3389/fmed.2020.557453

8. Guo Y, Liu Y, Lu J, et al. Development and validation of an early warning score (EWAS) for predicting clinical deterioration in patients with coronavirus disease 2019. medRxiv. 2020. doi:10.1101/ 2020.04.17.20064691

9. Ji D, Zhang D, Xu J, et al. Prediction for progression risk in patients with COVID-19 pneumonia: the CALL score. Clin Infect Dis. 2020;71(6):1393-1399. doi:10.1093/cid/ciaa414

10. Gidari A, De Socio GV, Sabbatini S, Francisci D. Predictive value of National Early Warning Score 2 (NEWS2) for intensive care unit admission in patients with SARS-CoV-2 infection. Infect Dis (Auckl). 2020;52(10):698-704. doi:10.1080/23744235.2020.1784457

11. Liang W, Liang H, Ou L, et al. Development and validation of a clinical risk score to predict the occurrence of critical illness in hospitalized patients with COVID-19. JAMA Intern Med. 2020;180 (8):1081-1089. doi:10.1001/jamainternmed.2020.2033

12. Matos J, Paparo F, Mussetto I, et al. Evaluation of novel coronavirus disease (COVID-19) using quantitative lung CT and clinical data: prediction of short-term outcome. Eur Radiol Exp. 2020;4(1):1-10. doi:10.1186/s41747-020-00167-0
13. Zou X, Li S, Fang M, et al. Acute physiology and chronic health evaluation II score as a predictor of hospital mortality in patients of Coronavirus Disease 2019. Crit Care Med. 2020;48(8):E657-E665. doi:10.1097/CCM.0000000000004411

14. Knight SR, Ho A, Pius R, et al. Risk stratification of patients admitted to hospital with covid-19 using the ISARIC WHO clinical characterisation protocol: development and validation of the $4 \mathrm{C}$ mortality score. BMJ. 2020;370:22. doi:10.1136/bmj.m3339

15. Charlson ME, Pompei P, Ales KL, MacKenzie CR. A new method of classifying prognostic comorbidity in longitudinal studies: development and validation. J Clin Epidemiol. 1987;40(5):373-383.

16. Elixhauser A, Steiner C, Harris DR, Coffey RM. Comorbidity measures for use with administrative data. Med Care. 1998;36(1):8-27. doi:10.1097/00005650-199801000-00004

17. Huntley AL, Johnson R, Purdy S, Valderas JM, Salisbury C. Measures of multimorbidity and morbidity burden for use in primary care and community settings: a systematic review and guide. Ann Fam Med. 2012;10(2):134-141. doi:10.1370/afm.1363

18. Gulbech Ording A, Toft Sørensen H. Concepts of comorbidities, multiple morbidities, complications, and their clinical epidemiologic analogs. Clin Epidemiol. 2013;5(1):199-203. doi:10.2147/CLEP. S45305

19. Monterde D, Cainzos-Achirica M, Cossio-Gil Y, et al. Performance of comprehensive risk adjustment for the prediction of in-hospital events using administrative healthcare data: the queralt indices. Risk Manag Healthc Policy. 2020;13:271-283. doi:10.2147/RMHP. S228415

20. Hick JL, Hanfling D, Wynia MK, Pavia AT. Duty to plan: health care, crisis standards of care, and novel Coronavirus SARS-CoV-2. NAM Perspectives. doi:10.31478/202003b

21. Mannucci E, Silverii GA, Monami M. Saturation of critical care capacity and mortality in patients with the novel coronavirus (COVID-19) in Italy. Trends Anaesth Crit Care. 2020;33:33-34. doi:10.1016/j.tacc.2020.05.002

22. Quan H, Sundararajan V, Halfon P, et al. Coding algorithms for defining comorbidities in ICD-9-CM and ICD-10 administrative data. Med Care. 2005;43(11):1130-1139. doi:10.1097/01. mlr.0000182534.19832.83

23. Moore BJ, White S, Washington R, Coenen N, Elixhauser A. Identifying increased risk of readmission and in-hospital mortality using hospital administrative data: the AHRQ Elixhauser Comorbidity Index. Med Care. 2017;55(7):698-705. doi:10.1097/ MLR.0000000000000735

24. Gasparini A. Package "Comorbidity"; 2020. Available from: https:// cran.r-project.org/web/packages/comorbidity/comorbidity.pdf. Accessed January 20, 2021.

25. Akaike H. A new look at the statistical model identification. IEEE Trans Automat Contr. 1974;19(6):716-723. doi:10.1109/TAC.19 74.1100705

26. Schwarz G. Estimating the dimension of a model. Ann Stat. 1978;6:461-464. doi:10.1214/aos/1176344136

27. Saito T, Rehmsmeier M. The precision-recall plot is more informative than the ROC plot when evaluating binary classifiers on imbalanced datasets. PLoS One. 2015;10(3):e0118432. doi:10.1371/journal. pone. 0118432

28. DeLong ER, DeLong DM, Clarke-Pearson DL. Comparing the areas under two or more correlated receiver operating characteristic curves: a nonparametric approach. Biometrics. 1988;44(3):837. doi:10.2307/ 2531595

29. $\mathrm{R}$ Core Team. R: A Language and Environment for Statistical Computing. Vienna, Austria: $\mathrm{R}$ Foundation for Statistical Computing; 2017. Available from: https://www.r-project.org. Accessed May 25, 2020.

30. Wang B, Jin S, Yan Q, et al. AI-assisted CT imaging analysis for COVID-19 screening: building and deploying a medical AI system. Appl Soft Comput. 2021;98:106897. doi:10.1016/j.asoc.2020.106897 
31. Li L, Qin $\mathrm{L}, \mathrm{Xu} \mathrm{Z}$, et al. Using artificial intelligence to detect COVID-19 and community-acquired pneumonia based on pulmonary CT: evaluation of the diagnostic accuracy. Radiology. 2020;296(2): E65-E71. doi:10.1148/radiol.2020200905

32. Abbas A, Abdelsamea MM, Gaber MM. Classification of COVID-19 in chest X-ray images using DeTraC deep convolutional neural network. Appl Intell. 2021;51(2):854-864. doi:10.1007/s10489-02001829-7

33. Li K, Fang Y, Li W, et al. CT image visual quantitative evaluation and clinical classification of coronavirus disease (COVID-19). Eur Radiol. 2020;30(8):4407-4416. doi:10.1007/s00330-020-06817-6

34. Tang Z, Zhao W, Xie X, et al. Severity assessment of Coronavirus Disease 2019 (COVID-19) using quantitative features from chest CT images. arXiv. 2020. doi:10.1088/1361-6560/abbf9e
35. Song J, Wang H, Liu Y, et al. End-to-end automatic differentiation of the coronavirus disease 2019 (COVID-19) from viral pneumonia based on chest CT. Eur J Nucl Med Mol Imaging. 2020;47 (11):2516-2524. doi:10.1007/s00259-020-04929-1

36. Ellinghaus D, Degenhardt F, Bujanda L, et al. Genomewide association study of severe Covid-19 with respiratory failure. $N$ Engl J Med. 2020;383(16):1522-1534. doi:10.1056/NEJMoa2020283

37. Stratton CW, Tang YW, Lu H. Pathogenesis-directed therapy of 2019 novel coronavirus disease. J Med Virol. 2021;93(3):1320-1342. doi: $10.1002 / \mathrm{jmv} .26610$

38. Capalbo C, Aceti A, Simmaco M, et al. The exponential phase of the covid-19 pandemic in central Italy: an integrated care pathway. Int J Environ Res Public Health. 2020;17(11):3792. doi:10.3390/ ijerph17113792
Risk Management and Healthcare Policy

\section{Publish your work in this journal}

Risk Management and Healthcare Policy is an international, peerreviewed, open access journal focusing on all aspects of public health, policy, and preventative measures to promote good health and improve morbidity and mortality in the population. The journal welcomes submitted papers covering original research, basic science, clinical \& epidemiological studies, reviews and evaluations,

\section{Dovepress}

guidelines, expert opinion and commentary, case reports and extended reports. The manuscript management system is completely online and includes a very quick and fair peer-review system, which is all easy to use. Visit http://www.dovepress.com/testimonials.php to read real quotes from published authors.

Submit your manuscript here: https://www.dovepress.com/risk-management-and-healthcare-policy-journal 